\title{
COLOR CONFINEMENT AND HADRONS IN QUANTUM CHROMODYNAMICS
}


This page intentionally left blank

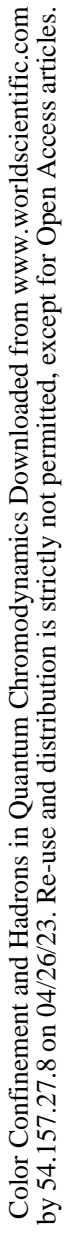




\title{
International Conference on COLOR CONFINEMENT AND HADRONS IN QUANTUM CHROMODYNAMICS
}

\author{
The Institute of Physical and \\ Chemical Research (RIKEN), Japan \\ $21-24$ July 2003
}

\section{Editors}

H Suganuma

N Ishii

M Oka

Tokyo Institute of Technology, Japan

H Enyo
RIKEN/RBRC, Japan

T Hatsuda

Tokyo University, Japan

T Kunihiro

Kyoto University, Japan

$K$ Yazaki

TWCU/RIKEN, Japan 


\section{Published by \\ World Scientific Publishing Co. Pte. Ltd. \\ 5 Toh Tuck Link, Singapore 596224}

USA office: Suite 202, 1060 Main Street, River Edge, NJ 07661

UK office: 57 Shelton Street, Covent Garden, London WC2H 9HE

\section{British Library Cataloguing-in-Publication Data \\ A catalogue record for this book is available from the British Library.}

\section{COLOR CONFINEMENT AND HADRONS IN QUANTUM CHROMODYNAMICS}

Proceedings of the International Conference on Confinement 2003

Copyright $\odot 2004$ by World Scientific Publishing Co. Pte. Ltd.

All rights reserved. This book, or parts thereof, may not be reproduced in any form or by any means, electronic or mechanical, including photocopying, recording or any information storage and retrieval system now known or to be invented, without written permission from the Publisher.

For photocopying of material in this volume, please pay a copying fee through the Copyright Clearance Center, Inc., 222 Rosewood Drive, Danvers, MA 01923, USA. In this case permission to photocopy is not required from the publisher.

ISBN 981-238-781-1 


\title{
ORGANIZING COMMITTEE
}

\author{
H. Suganuma (IRCS, Tokyo Institute of Technology, Chair) \\ H. Enyo (RIKEN/RIKEN-BNL Research Center) \\ T. Hatsuda (The University of Tokyo) \\ N. Ishii (RIKEN/Tokyo Institute of Technology) \\ T. Kunihiro (YITP, Kyoto University) \\ M. Oka (Tokyo Institute of Technology) \\ K. Yazaki (Tokyo Woman's Christian Univ./RIKEN)
}

\section{HOST INSTITUTE}

Interactive Research Center of Science (IRCS),

Tokyo Institute of Technology

The Institute of Physical and Chemical Research (RIKEN) 
This page intentionally left blank

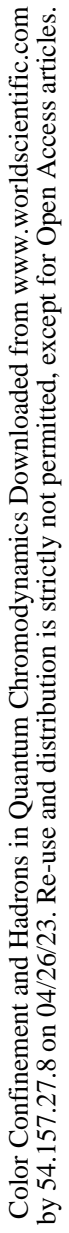




\section{PREFACE}

The International Conference on "Color Confinement and Hadrons in Quantum Chromodynamics" (Confinement 2003) was held from July 21 to 24 in 2003 at the Institute of Physical and Chemical Research (RIKEN) with the host of Interactive Research Center of Science (IRCS) at Tokyo Institute of Technology. This is the successor of the International Symposia on "Color Confinement and Hadrons" (Confinement '95) and "Quantum Chromodynamics and Color Confinement" (Confinement 2000) both held in Osaka.

Quantum chromodynamics (QCD) has been established as the fundamental theory of the strong interaction three decades ago. Nevertheless, there remain lots of interesting problems related to nonperturbative dynamics in the infrared region of QCD such as color confinement, dynamical chiral-symmetry breaking and nontrivial topologies.

To understand the nonperturbative physics of $\mathrm{QCD}$, numerous theoretical studies have been done with various effective models and mathematical approaches. In particular, the lattice QCD Monte Carlo simulation has been established as a powerful method for the analysis of nonperturbative QCD. From the experimental viewpoint, QCD physics encounters a new phase owing to the interesting experimental data at the RHIC project in Brookhaven National Laboratory.

The purpose of the present Conference is to summarize the current status of nonperturbative QCD in mathematical physics, lattice QCD physics and quark-hadron physics, and to promote the mutual understanding among these fields, as a necessary step for the global understanding of nonperturbative QCD.

The subjects covered in this Conference are as follows:

- Quark Confinement Mechanism

- Dynamical Chiral-Symmetry Breaking

- Topologies in QCD (Instantons, Monopoles and Vortices)

- Confinement in SUSY QCD

- Nonperturbative Analysis ( $1 / N_{c}$, ladder QCD, AdS/CFT)

- QCD Phase Transition at Finite Temperature and Density

- Quark Gluon Plasma

This Conference consists of plenary sessions, parallel sessions and a poster session. The number of participants was 131 in which 64 were from overseas from 18 different countries. For the active communication of participants, 
the poster session was held combined with a reception party. We had lively and active discussions and useful communications among the participants from different fields of physics.

These proceedings consist of 28 papers for the invited talks at the plenary sessions, 20 papers for the selected talks at the parallel sessions and 33 one-page abstracts for selected posters. For the plenary talks, we have sorted the papers into 6 categories in terms of their theme of physics. In each category, the papers are arranged in the order of the presentation at the Conference. For the parallel and the poster presentations, the papers are arranged in alphabetical order of the presenter's family name.

We believe that these proceedings will be useful for the global understanding of the current studies on the nonperturbative aspects of QCD from wide viewpoints of mathematical physics, lattice QCD physics and quark-hadron physics.

The Conference organizing committee would like to express its cordial thanks to all the participants, the speakers, and the chairpersons for making the Conference very successful. This Conference was partially supported by Inoue Foundation for Science in Japan.

We are extremely grateful to Ms. Noriko Kiyama at RIKEN for her devoted works for all aspects on the Conference. We acknowledge the members of TokyoTech theory group and RIKEN for their help during the Conference. We particularly thank Drs. T.T. Takahashi, T. Doi, H. Iida, $K$. Tanida and Y. Watanabe for their devoted helps.

We thank Prof. K.-I. Aoki for his wonderful photographs at Confinement 2003. The cover design is based on the recent lattice QCD results for the three-quark flux-tube formation by Drs. H. Ichie, V. Bornyakov, T. Streuer and G. Schierholz. We acknowledge them for their help in designing the proceedings cover.

Finally, H.S. thanks Mrs. Aigo Suganuma for many helps on the preparation of the Conference.

\section{The Organizing Committee of Confinement 2003}

Hideo Suganuma (IRCS, Tokyo Institute of Technology, Chair) Hideto Enyo (RIKEN/RIKEN-BNL Research Center)

Tetsuo Hatsuda (The University of Tokyo)

Noriyoshi Ishii (RIKEN/Tokyo Institute of Technology)

Teiji Kunihiro (YITP, Kyoto University)

Makoto Oka (Tokyo Institute of Technology)

Koichi Yazaki (Tokyo Woman's Christian University/RIKEN) 


\section{CONTENTS}

Preface vii

Supersymmetric QCD

Supersymmetric Gluodynamics and its Nonsupersymmetric Cousins:

Planar Equivalence

M. Shifman, A. Armoni and G. Veneziano

Non-Abelian Superconductor and QCD:

Lessons from Supersymmetric Theories

28

K. Konishi

Confinement Mechanism in QCD

Confinement of Color: Recent Progress

A. Di Giacomo

Consistent Definitions of Flux and Electric and Magnetic Current in Abelian Projected SU(2) Lattice Gauge Theory

R.W. Haymaker and T. Matsuki

Connecting Short to Long Scales in the Confining Vacuum

E.T. Tomboulis

SO(3) vs. SU(2) Yang-Mills Theory on the Lattice:

An Investigation at Non-Zero Temperature

A. Barresi, G. Burgio and M. Müller-Preussker

On the Vacuum of Full QCD

G. Schierholz

Branes in Lattice SU(2) Gluodynamics 
Are Glueballs Knotted Closed Strings ?

A.J. Niemi

Vacuum Condensates, Effective Gluon Mass and Color Confinement 140 K.-I. Kondo

Adventures in Coulomb Gauge

J. Greensite and $\check{S}$. Olejnik

Conformal Aspects of QCD

S.J. Brodsky

Non-Perturbative Renormalization Group Analysis

for Dynamical Chiral Symmetry Breaking in QCD

K.-I. Aoki

Light Mesons and Infrared Behavior of

the Running Coupling Constant in QCD

M. Baldicchi and G. Prosperi

Renormalon Cancellation and Perturbative QCD Potential

as a Coulomb + Linear Potential

Y. Sumino

Infrared Limit and Mass Gap in QCD

D. Zwanziger

Lattice QCD for Quark-Hadron Physics

Excitations of the Static Quark-Antiquark System

in Several Gauge Theories

K.J. Juge, J. Kuti and C. Morningstar

QCD String Formation and the Casimir Energy

K.J. Juge, J. Kuti and C. Morningstar

Y-type Flux-Tube Formation and Gluonic Excitations in Baryons:

From QCD to Quark Model

H. Suganuma, H. Ichie and T.T. Takahashi 
Lattice Calculation of the Lowest Order Hadronic Contribution to the Muon Anomalous Magnetic Moment:

An Update with Kogut-Susskind Fermions

T. Blum

Hot and Dense QCD

Deconfinement in QCD and in Nuclear Collisions

H. Satz

Confronting Lattice Results on the Transition

from the Hadron Gas to the Quark-Gluon Plasma

with Hadronic Resonance Gas Models

F. Karsch

Jet Tomography of Hot and Dense Matter

X.-N. Wang

A New Method for Simulating QCD at Finite Density

J. Nishimura

Dense Quark Matter in Compact Stars

M. Alford

Thermal Phase Transition of Dense QCD

T. Matsuura, T. Hatsuda, K. Iida and G. Baym

Recent Experiments on QCD

(Quark Gluon Plasma \& Penta-Quark System)

Experimental Results from RHIC

Y. Akiba

Experimental Evidence for Penta-Quark System

T. Nakano for the LEPS Collaboration 
Calculation of Finite Size Effects on the Nucleon Mass in Unquenched QCD using Chiral Perturbation Theory

A. Ali Khan, T. Bakeyev, M. Göckeler, T.R. Hemmert,

R. Horsley, A.C. Irving, D. Pleiter, P.E.L. Rakow,

G. Schierholz and H. Stüben

The Factorization Method for Simulating

Systems with a Complex Action

J. Ambjørm, K.N. Anagnostopoulos, J. Nishimura and J.J.M. Verbaarschot

Gluodynamics in External Field:

A Test of the Dual Superconductor Picture

M. Chernodub

The Study of Chiral Restoration using the Quark-Gluon Mixed Condensate $g\left\langle\bar{q} \sigma_{\mu \nu} G_{\mu \nu} q\right\rangle$ in Lattice QCD at Finite Temperature

T. Doi, H. Suganuma, M. Oka and N. Ishii

The 2PPI Expansion: Dynamical Mass Generation and Vacuum Energy

D. Dudal, H. Verschelde, R.E. Browne and J.A. Gracey

Numerical Study of the Equation of State for Two Flavor QCD at Finite Density

S. Ejiri, C.R. Allton, S.J. Hands, O. Kaczmarek,

F. Karsch, E. Laermann and C. Schmidt

Topology and Chirality Mixing Interaction in QCD

P. Faccioli and T.A. DeGrand

The Study of the Continuum Limit of the Supersymmetric

Ward-Takahashi Identity for $N=1$ Super Yang-Mills Theory

A. Feo

When is the Deconfinement Phase Transition Universal ?

K. Holland, M. Pepe and U.-J. Wiese

Properties of Thermal Glueballs

N. Ishii and H. Suganuma 
Features of SU(N) Gauge Theories

B. Lucini, M. Teper and U. Wenger

Monopoles in Real Time for Classical U(1) Gauge Field Theory

T. Biró, H. Markum and R. Pullirsch

Charmonium Correlators at Finite Temperature

in Quenched Lattice QCD

H. Matsufuru, T. Umeda and K. Nomura

Momentum Distribution of $J / \psi$ 's Produced at RHIC

F.O. Durães, F.S. Navarra and M. Nielsen

Open Charm Production through $J / \psi-\pi$ Collisions

M. Nielsen, F.S. Navarra and F.O. Durães

Effective String Picture for Confining Gauge Theories at Finite Temperature

M. Caselle, M. Panero and M. Hasenbusch

Vacuum Condensates and Dynamical Mass Generation in Euclidean Yang-Mills Theories

D. Dudal, H. Verschelde, V.E.R. Lemes, M.S. Sarandy, R.F. Sobreiro, S.P. Sorella, M. Picariello, A. Vicini and J.A. Gracey

Y-type Flux-Tube Formation in Baryons

T.T. Takahashi, H. Suganuma and H. Ichie

Motion of Quarks inside Heavy Flavor Hadrons and the QCD Equations of Motion

K. Tanaka

Kinks in Time and Their Relation to Confinement

J.E. Wang

Poster Session Presentations

Conference Program

Participant List 